\title{
LIE SIMPLICITY OF A SPECIAL CLASS OF ASSOCIATIVE RINGS II $\left({ }^{1}\right)$
}

\author{
BY \\ WILLARD E. BAXTER( $\left.{ }^{2}\right)$
}

Introduction. Let $A$ be a simple associative ring with an involution defined on it; that is, a mapping of $A$ onto $A$, defined by $a \rightarrow a^{*}$, with the following properties:

(i) $\left(a^{*}\right)^{*}=a$; (ii) $(a+b)^{*}=a^{*}+b^{*}$; (iii) $(a b)^{*}=b^{*} a^{*}$.

Recently I. N. Herstein [4] has investigated $S=\left\{x \in A \mid x^{*}=x\right\}$ and $K=\left\{k \in A \mid k^{*}=-k\right\}$, the sets of symmetric and skew symmetric elements respectively.

The set $K$ has the property that if $k$ and $l$ are in $K$, then $k l-l k$ is again in $K$. Thus, $K$ is a Lie subring of $A$ under the Lie multiplication $[x, y]$ $=x y-y x$ defined for all $x, y \in A$. An additive subgroup $U$ of $K$ is said to be a Lie ideal of $K$ if, in addition, $u k-k u \in U$ for all $u \in U$ and $k \in K$.

Of particular interest is the Lie ideal $[K, K]$, the additive subgroup generated by all elements of form $k l-l k, k$ and $l$ in $K$. Herstein [4, Theorem 26] has shown the Lie structure of $K$ to be as follows:

TheOREM 1. Let $A$ be a simple ring of characteristic $\neq 2$, with an involution and suppose that either $Z=(0)$, or that $A$ is more than 16-dimensional over $Z$, its center; if $K$ is the set of skew elements of $A$ then every Lie ideal, $U$, of $K$ must satisfy

(1) either $U \subset Z$ or (2) $U \supset[K, K]$.

In this paper we investigate the Lie structure of $[K, K]$. The main result states that if $A$ is as above, then $[K, K]$ modulo its intersection with $Z$ is a simple Lie ring.

To prove this result we shall make use of the following lemmas:

Lemma 1. Let $A$ be a simple ring of characteristic not 2. Suppose that $t \in A$ is such that $t(t a-a t)=(t a-a t) t$ for all $a \in A$, then $t \in Z$.

The proof of this lemma is given in Herstein [4, p. 641].

LEMMA 2. Let $A$ be a simple ring, and

(i) if $z \neq 0 \in S \cap Z$, then $z K=K$,

(ii) if $z \neq 0 \in K \cap Z$, then $z K=S$.

Presented to the Society April 21, 1956; received by the editors August 16, 1956.

(1) The results of this paper comprise the final portion of a thesis, which was presented to the Faculty of the Graduate School of the University of Pennsylvania in partial fulfillment of the requirements for the degree of Doctor of Philosophy.

(2) The author wishes to take this opportunity to thank Professor I. N. Herstein who suggested this problem. His valuable assistance and kind encouragement are greatly appreciated. 
The proof of this lemma is a trivial consequence of $\left(z^{*}\right)^{-1}=\left(z^{-1}\right)^{*}$.

In order to simplify the stating of theorems, from this point on we shall assume that $A$ is a simple associative ring, of characteristic $\neq 2$, whose center $Z=(0)$, or whose dimension over $Z$ is greater than 16 , unless otherwise indicated.

Definition. By $\bar{U}=U^{-}$we mean the subring generated by the elements of $U$.

It has been proven that if $A$ is as above then $\bar{K}=\bar{S}=A$, cf. $[4$, Theorems 9 and 15]. Using this we are able to prove the following lemma.

Lemma 3. $[K, K] \neq(0)$.

Proof. Assume that $[K, K]=(0)$. Fix $k \in K$. Then $k l-l k=0$ for all $l \in K$. Hence, $k l^{\prime}=l^{\prime} k$ for all $l^{\prime} \in \bar{K}$. However, $\bar{K}=A$. Thus, $k \in Z$. Since this is true for all $k \in K$, then $K \subset Z$. Now on the one hand if $K=(0)$ then $A=S$ and since $[S, S] \subset K=(0)$ we have that $A$ is commutative, a contradiction. On the other hand, if $K \neq(0)$ then let $\lambda \neq 0 \in K \cap Z$. Then $\lambda S=K$ and since $Z$ is a field, $\lambda^{-1}$ exists and therefore $\lambda^{-1}(\lambda S)=S \subset Z$. Hence, since $A=S+K$ then $A \subset Z$, a contradiction.

Definition. The lie product of $A$ effected by an element $a \in A$ will be denoted by $D_{a}(x)$; that is, $D_{a}(x)=a x-x a$. We further define for all $n \geqq 1$, $D_{a}^{(n)}(x)=D_{a}\left(D_{a}^{(n-1)}(x)\right)$ and $D_{a}^{(1)}(x)=D_{a}(x)$.

Lemma 4. Let $B$ be any additive subgroup of $A$ such that $\bar{B}=A$ and let $U$ be any Lie ideal of $B$, then

(i) $[B, \bar{U}] \subset \bar{U}$ and

(ii) if there exists $u^{\prime} \in \bar{U}$ such that $A u^{\prime} \subset \bar{U}$, then either $u^{\prime}=0$ or $\bar{U}=A$.

Proof. (i) Let $b \in B, u_{1}, \cdots, u_{n} \in U$, then $D_{b}\left(u_{1} \cdots u_{n}\right)=\sum_{i} u_{1} \cdots u_{i-1}$ $\cdot D_{b}\left(u_{i}\right) u_{i+1} \cdots u_{n}$ and for each $i, D_{b}\left(u_{i}\right) \in U$. Hence, $D_{b}\left(u_{1} \cdots u_{n}\right) \in \bar{U}$; that is, $\left[B, U^{n}\right] \subset \bar{U}$ for all $n$. So, $[B, \bar{U}] \subset \bar{U}$.

(ii) Suppose there exists $u^{\prime} \in \bar{U}$ such that $a u^{\prime} \in \bar{U}$ for all $a \in A$. Let $b \in B$, then $D_{b}\left(a u^{\prime}\right) \in \bar{U}$ by the above. That is, $b\left(a u^{\prime}\right)-\left(a u^{\prime}\right) b \in \bar{U}$ and since $b a u^{\prime} \in \bar{U}$ by hypothesis then $a u^{\prime} b \in \bar{U}$. Suppose for any $n-1$ elements of $B$, $a u^{\prime} b_{1} \cdots b_{n-1} \in \bar{U}$, and let $b_{n} \in B$. Then $b_{n}\left(a u^{\prime} b_{1} \cdots b_{n-1}\right)-\left(a u^{\prime} b_{1} \cdots b_{n-1}\right) b_{n}$ $\in \bar{U}$. However, since by assumption $b_{n} a u^{\prime} b_{1} \cdots b_{n-1} \in \bar{U}$ then $a u^{\prime} b_{1} \cdots b_{n}$ $\in \bar{U}$; that is, $A u^{\prime} \bar{B} \subset \bar{U}$. Thus, since $\bar{B}=A$ we have $A u^{\prime} A \subset \bar{U}$. But, since $A$ is simple, then either $A u^{\prime} A=(0)$ in which case $u^{\prime}=0$, or $A u^{\prime} A=A$ in which case $A=\bar{U}$.

THEOREM 2. Let $U$ be a Lie ideal of $K$; then either

(i) $\bar{U}=A$ or (ii) $u^{2} v=v u^{2}$ for all $u, v \in U$.

Proof. If $s \in S, u \in U$, then

(I) $u^{2} s-s u^{2}=u(u s+s u)-(u s+s u) u \in U$ since $u s+s u \in K$.

If $k \in K, u \in U$ then

(II) $u^{2} k-k u^{2}=u(u k-k u)+(u k-k u) u \in \bar{U}$ since $u k-k u \in U$. Since $A$ $=S+K$, then combining (I) and (II) we have for all $a \in A$ 


$$
u^{2} a-a u^{2} \in \bar{U} \text {. }
$$

If $w \in \bar{U}$, then $(a w) u^{2}-u^{2}(a w)=a\left(w u^{2}-u^{2} w\right)+\left(a u^{2}-u^{2} a\right) w$. The left side is in $\bar{U}$ and $\left(a u^{2}-u^{2} a\right) w$ is also in $\bar{U}$ since $a u^{2}-u^{2} a$ and $w$ are in $\bar{U}$; thus, for all $a \in A, w \in \bar{U}$, and $u \in U, a\left(w u^{2}-u^{2} w\right) \in \bar{U}$. That is,

$$
A\left(w u^{2}-u^{2} w\right) \subset \bar{U} .
$$

By Lemma 4(ii), since $\bar{K}=A$, either $\bar{U}=A$ or $w u^{2}-u^{2} w=0$. In particular, choosing $w \in U$ we have either $A=\bar{U}$ or $u^{2} v=v u^{2}$ for all $u, v \in U$.

The subring $[K, K]$. We are now in a position to investigate the subring $[K, K]$. In the proof of the theorems concerning the Lie ideals of $K$, one of the main results used is that $\bar{K}=A$. Thus, one sees the necessity of proving a similar result for $[K, K]-$ if one is to investigate the Lie simplicity of $[K, K]$.

In order to show that $[K, K]^{-}=A$, it is necessary to consider two distinct cases; namely:

(i) $\lambda^{*}=\lambda$, for all $\lambda \in Z$; in which case the involution is said to be of the first kind.

(ii) $\lambda^{*}=-\lambda$, for some $\lambda \neq 0 \in Z$; in which case the involution is said to be of the second kind.

In the case of involution of the second kind, we make use of the following theorem which was proven by Herstein [4]. We give here a new proof.

THEOREM 3. If $A$ has an involution of the second kind and if $U$ is a Lie ideal of $K$ such that $u^{2} \in Z$ for all $u \in U$, then $U \subset Z$.

Proof. Since $A$ has an involution of the second kind, then $A$ is of dimension greater than 16 over $Z \neq 0$, and by Lemma 2(ii), $A=K+q K$ where $q \in Z$ and $q^{*}=-q$. Consider $[Z U, A]=[Z U, K+q K]=[Z U, K]+[Z U, q K] \subset Z U$. Therefore, $Z U$ is a Lie ideal of $A$. Thus, by Herstein [2], either $Z U \subset Z$ or $Z U \supset[A, A]$. If on the one hand $Z U \subset Z$, then $U \subset Z$. If on the other hand $Z U \supset[A, A]$, then since $u^{2} \in Z$ for all $u \in U$, it follows that $a^{2} \in Z$ for all $a \in[A, A]$. This means that $A$ satisfies a polynomial identity (cf. I. Kaplansky [5]) and, being simple, it must be finite dimensional over $Z$. For this particular identity it is known that $A$ is at most 4-dimensional over $Z$ (Kaplansky [5]). This is a contradiction since $A$ is more than 16-dimensional over $Z$.

Theorem 4. The subring $[K, K]^{-}$, generated by $[K, K]$, is $A$.

Proof. In Theorem 2 we have shown that either $[K, K]^{-}=A$ or $u^{2} v=v u^{2}$ for all $u, v \in[K, K]$. The claim is that when $u^{2} v=v u^{2}$ for all $u, v \in[K, K]$ then $[K, K]=(0)$, which by Lemma 3 is a contradiction.

Let $u \in[K, K], k \in K$. Then, since $u k-k u \in[K, K]$, we have $u^{2}(u k-k u)$ $=(u k-k u) u^{2}$. Therefore, $u^{2}\left[u^{2} k-k u^{2}\right]=\left[u^{2} k-k u^{2}\right] u^{2}$. Let $s \in S$. Then $u^{2} s-s u^{2} \in[K, K]$ and so $u^{2}\left[u^{2} s-s u^{2}\right]=\left[u^{2} s-s u^{2}\right] u^{2}$. Since $A=S+K$, then $u^{2}\left[u^{2} a-a u^{2}\right]=\left[u^{2} a-a u^{2}\right] u^{2}$ for all $a \in A$. Hence, by Lemma $1, u^{2} \in Z$ for all 
$u \in[K, K]$. Let $u$ and $v \in[K, K]$. Then $(u+v)^{2}-u^{2}-v^{2} \in Z$. Hence, $u v+v u$ $\in Z$ for all $u, v \in[K, K]$. If $u^{2}=0$ for all $u \in[K, K]$ then, by Herstein [4], $[K, K]=(0)$, a contradiction.

So suppose that $Z \neq(0)$ and $u^{2} \neq 0$ for some $u \in[K, K]$. At this point it is necessary to consider the types of involution separately.

If, on the one hand, the involution is of the second kind then, by Theorem $3,[K, K] \subset Z$. Hence $[[K, K],[K, K]]=(0)$. But, by Herstein $[4],[[K, K]$, $[K, K]]=[K, K]$ and therefore $[K, K]=(0)$, a contradiction.

If, on the other hand, the involution is of the first kind then, by Herstein [4, proof of Theorem 24], it follows that there exists $u, v \in[K, K]$ such that $u, v$, and $u v-v u$ form a basis of $[K, K]$ over $Z$ and such that $u^{2} \neq 0, v^{2} \neq 0$ and $u v+v u=0$. As in Herstein's proof we define $N(u)=\{x \in K \mid x u=u x\}$ and similarly define $N(v)$. Clearly, $W=N(u) \cap N(v)=\{x \in K \mid[x,[K, K]]=(0)\}$. The claim is that $W \cap[K, K]=(0)$, for suppose that there exists $a \neq 0 \in[K, K]$ such that $[a,[K, K]]=(0)$. Now, $a=\alpha u+\beta v+\delta(u v-v u)$ where $\alpha, \beta$ and $\delta$ are in $Z$ and not all zero. Now, $a u=u a$ implies that $\beta v u+\delta(u v-v u) u=\beta u v$ $+\delta u(u v-v u)$. Collecting together, we have

$$
\beta(v u-u v)=\delta[u(u v-v u)-(u v-v u) u] .
$$

Since $u v=-v u$ and $u^{2} \in Z$, this latter reduces to

$$
\beta(v u-u v)=4 \delta u^{2} v .
$$

Since $v$ and $u v-v u$ are linearly independent over $Z$ and $u^{2} \neq 0 \in Z$, this last equation is possible if, and only if, $\beta=\delta=0$. Thus, $a=\alpha u$. Since $a \in U$, then $a v=v a$; that is, $\alpha(u v-v u)=0$ and thus $\alpha=0$, which is a contradiction to $a \neq 0$. Hence, $W \cap[K, K]=(0)$. However, by Herstein $[4$, proof of Theorem $24]$, it follows that $W \neq(0), W$ is a Lie ideal of $K$, and $W \subset Z$. This is a contradiction since $K \cap Z=(0)$.

Therefore, in either case, if $u^{2} v=v u^{2}$ for all $u, v \in[K, K]$ we have a contradiction. Hence, $[K, K]^{-}=A$.

The Lie ideals of $[K, K]$.

Lemma 5. Let $U \neq(0)$ be a Lie ideal of $[K, K]$ and $W=\{x \in A \mid x U=(0)\}$ : then $W=(0)$.

Proof. Let $w \in W, k \in[K, K]$ and $u \in U$; then $w(u k-k u)=0$. However, $w u k=0$ and therefore $w k u=0$; that is, $W[K, K] \subset W$. Hence, $W[K, K]-\subset W$ and, by Theorem 4, this means that $W A \subset W . W$ is also a left ideal of $A$ and so $W$ is a two-sided ideal of $A$. Since $A$ is simple, either $W=A$ or $W=(0)$. If $W=A$, then $A U=(0)$ which in a simple ring forces $U=(0)$, a contradiction. Hence $W=(0)$.

Definition. If $U$ is a lie ideal of $[K, K]$, we define $T=T(U)=\{t \in K \mid$ $[t, K] \subset U\}$.

In the study of the Lie ideals of $A,[A, A]$ and $K$, sets similar to $T$ are 
considered. The properties of $T$ which play an important role in the proof of the theorems which follow are collected together in the following lemma:

Lemma 6. For a given Lie ideal $U$ of $[K, K], T=T(U)$ has the following properties:

(i) $[T,[K, K]] \subset T$; (ii) $[U, U] \subset T$; (iii) $t^{2} s-s t^{2} \in U$ for all $t \in T, s \in S$; and (iv) if $2 w \in T$ then $w \in T$.

Proof. (i) Let $t \in T, l \in[K, K]$ and $k \in K$. Then

$$
\begin{gathered}
{[[t, l], k]=[t,[l, k]]+[[t, k], l] \subset U+[U,[K, K]] \subset U \text {; that is }} \\
{[T,[K, K]] \subset T .}
\end{gathered}
$$

(ii) Let $u, v \in U$ and $k \in K$. Then $[[u, v], k]=[u,[v, k]]+[[u, k], v] \in U$. Hence, $[U, U] \subset T$.

(iii) Let $t \in T \subset K$ and $s \in S$. Then $t s+s t \in K$ and so $t^{2} s-s t^{2}=t(t s+s t)$ $-(t s+s t) t \in U$.

(iv) Let $2 w \in T$. Then $[2 w, K]=[w, 2 K]$ and since the characteristic $\neq 2$, then $K=2 K$. Hence, $w \in T$.

We now have the necessary tools to attack the problem of the simplicity of $[K, K]$. We first prove some general theorems about Lie ideals of $[K, K]$. After this, however, it will be necessary to divide the argument into two cases; namely, involutions of the first and second kind respectively.

Lemma 7. If $U$ is a Lie ideal of $[K, K]$ such that $u^{2}=0$ for all $u \in U$, then $U=(0)$.

Proof. Since $u^{2}=0$ for all $u \in U$, then $(u+v)^{2}-u^{2}-v^{2}=0$ for all $u, v \in U$. That is, $u v+v u=0$. Since $u v-v u \in U$, we have $2 u v \in U$ for all $u, v \in U$. Thus,

(I) $2(u v) w+w(2 u v)=0$ for all $u, v, w \in U$. Also, $v w+w v=0$ and therefore $2 u v w+2 u w v=0$. Hence,

(II) $2 u v w-2 w u v=0$.

Adding (I) and (II) we have $4 u v w=0$, and therefore $u v w=0$ since the characteristic $\neq 2$. That is, $u v U=(0)$ and thus, by Lemma $5, u v=0$. Therefore, $u U=(0)$ and hence $u=0$ for all $u \in U$. This completes the proof.

Lemma 8. Let $U$ be a proper Lie ideal of $[K, K]$ such that $u^{2} \in Z \neq(0)$ for all $u \in U$; then $U \subset Z$.

Proof. Let $u \in U, k \in K$; then $u(u k-k u)-(u k-k u) u \in U$ and since $u^{2} \in Z$, then $2 u(u k-k u) \in U$. However, since $2 K=K$ then $u\left(u k^{\prime}-k^{\prime} u\right) \in U$ for all $u \in U, k^{\prime} \in K$. Setting $k^{\prime}=u k-k u$ it then follows, by the above, that $u^{2}\left(u k^{\prime \prime}-k^{\prime \prime} u\right) \in U$ for all $k^{\prime \prime} \in K$.

Since $u^{2} \in Z$, then $u\left(u^{2} k\right)-\left(u^{2} k\right) u \in U$ for all $u \in U, k \in K$. Now, if $u^{2} \neq 0$ then $u^{2} K=K$ and hence $u k^{\prime}-k^{\prime} u \in U$ for all $k^{\prime} \in K$. The claim is that if $u^{2}=0$ then $u k-k u \in U$ for all $k \in K$ also. If this is the case, then $U$ is a Lie ideal of $K$ and therefore, by Theorem 1 , either $U \subset Z$ or $[K, K] \subset U$. The latter is 
impossible if $U$ is a proper Lie ideal of $[K, K]$. Hence, $U \subset Z$. Thus, it remains to show that $u^{2}=0$ implies that $u k-k u \in U$ for all $k \in K$. There exists $v \in U$ such that $v^{2} \neq 0$ otherwise, by Lemma $7, U=(0)$. Thus, without loss of generality, we may assume that $(u+v)^{2} \neq 0$ for otherwise if both $(u+v)^{2}=0$ and $(u-v)^{2}=0$ then

$$
0=(u+v)^{2}+(u-v)^{2}=2 u^{2}+2 v^{2}
$$

and therefore $v^{2}=0$ which is contrary to hypothesis. Since, $w^{2}(w k-k w) \in U$ for all $w \in U, k \in K$, then in particular:

$$
(u+v)^{2}[(u+v) k-k(u+v)] \in U .
$$

Hence, $\quad(u+v)\left[(u+v)^{2} k\right]-\left[(u+v)^{2} k\right](u+v) \in U$. However, since $(u+v)^{2}$ $\neq 0 \in Z$ then $(u+v)^{2} K=K$ and therefore,

$$
(u+v) k^{\prime}-k^{\prime}(u+v) \in U \text { for all } k^{\prime} \in K .
$$

That is,

$$
u k^{\prime}-k^{\prime} u+\left(v k^{\prime}-k^{\prime} v\right) \in U \text { for all } k^{\prime} \in K .
$$

However, since $v^{2} \neq 0$, by the preceding we have seen that $v k^{\prime}-k^{\prime} v \in U$ for all $k^{\prime} \in K$. Hence,

$$
u k^{\prime}-k^{\prime} u \in U \text { for all } k^{\prime} \in K .
$$

Thus, we have proven our contention.

Lemma 9. If $U$ is any subset of an arbitrary ring $A$ such that $\bar{U}=A$, then $[U, A]=[A, A]$.

Proof. Let $u_{1}, u_{2}, \cdots, u_{n} \in U$ and $a \in A$; then

$$
D_{a}\left(u_{1}, \cdots u_{n}\right)=\sum_{i} D_{u_{i}}\left(u_{i+1} \cdots u_{n} a u_{1} \cdots u_{i-1}\right) .
$$

Hence, $\left[U^{n}, A\right] \subset[U, A]$ for any $n$. Thus, $[\bar{U}, A] \subset[U, A]$; that is, $[U$, $A]=[A, A]$.

Using this lemma we are able to gain valuable information about $[A, A]$.

LEMMA 10. If $A$ is a simple ring with $Z=(0)$ or of dimension greater than 16 over $Z$ with an involution defined on it, then $[K, K]=[S, S]$ and $[A, A]$ $=[S, K]+[K, K]$.

Proof. By Herstein [4], $\bar{K}=A$ and $\bar{S}=A$; therefore, by Lemma $9,[K, A]$ $=[A, A]$ and $[S, A]=[A, A]$. These relations yield

(I) $[K, S+K]=[S+K, S+K]$ and

(II) $[S, S+K]=[S+K, S+K]$ respectively.

Due to the fact that $S \cap K=(0)$, from (I) we see that $[K, K] \supset[S, S]$ and from (II) that $[S, S] \supset[K, K]$. Hence, $[K, K]=[S, S]$, and therefore, $[A, A]=[S, K]+[K, K]$. 
We are now in a position to investigate where the Lie ideals of $[K, K]$ are situated. The key to this problem is:

Lemma 11. If $U$ is a proper Lie ideal of $[K, K]$, then either $\bar{U}=A$ or $[U, U] \subset Z$.

Proof. Let $w \in T \cap U$; then by Lemma $6, w^{2} s-s w^{2} \in U$ for all $s \in S$, and $w^{2} k-k w^{2}=w(w k-k w)+(w k-k w) w \in \bar{U}$ for all $k \in K$. Thus, for all $w \in T \cap U$ and $a \in A, w^{2} a-a w^{2} \in \bar{U}$. If $t \in T$ and $u \in U$, then $t u-u t \in T \cap U$; that is, $[T, U] \subset T \cap U$, and so, by the above, for all $v \in[T, U], a \in A, D_{v}{ }^{2}(a) \in \bar{U}$. Substituting $a u$ for $a, u \in U$, we have $D_{v^{2}}(a u)=D_{v^{2}}(a) u+a D_{v^{2}}(v) \in \bar{U}$. However, $D_{v^{2}}(a) u \in \bar{U}$ and so for all $a \in A, v \in[T, U]$ and $u \in U$ we have

$$
a\left(v^{2} u-u v^{2}\right) \in \bar{U} \text {. }
$$

However, by setting $B=[K, K]$ in Lemma 4 , we have either $\bar{U}=A$ or $u v^{2}-v^{2} u=0$ for all $u \in U, v \in[T, U]$.

Hence, it remains to show that $D_{v^{2}}(u)=0$ implies that $[U, U] \subset Z$. Since $v^{2} s-s v^{2} \in U$, then $D_{v^{2}}^{(2)}(s)=0$ for all $s \in S, v \in[T, U]$. Also, since $D_{v^{2}}(v k-k v)$ $=0$ for all $k \in K$, it follows that $D_{v^{2}}^{(2)}(k)=0$. Therefore, by Lemma $1, v^{2} \in Z$ for all $v \in[T, U]$.

On the one hand, suppose that $v^{2}=0$ for all $v \in[T, U]$; then, by Lemma 7 , we have, since $[T, U]$ is a Lie ideal of $[K, K]$, that $[T, U]=(0)$. Thus, $t u-u t=0$ for all $t \in T, u \in U$. Hence, $t\left(t^{2} s-s t^{2}\right)=\left(t^{2} s-s t^{2}\right) t$ and therefore $t^{2}$ commutes with $t^{2} s-s t^{2}$. Also, $t(t k-k t)=(t k-k t) t$ and therefore $t^{2}$ also commutes with $t^{2} k-k t^{2}$. Thus, as before, $t^{2} \in Z$ for all $t \in T$. In particular, $u^{2} \in Z$ if $u \in[U, U] \subset T$. Thus, by Lemma 7 and Lemma $8,[U, U] \subset Z$.

On the other hand, suppose that $v^{2} \in Z$ for all $v \in[T, U]$ and that $w^{2} \neq 0$ for some $w \in[T, U]$. By Lemma $8,[T, U] \subset Z$. Let $t \in T, u \in U$; then $t(t u-u t) t \in T$. Whence, $t(t u-u t) t u-u t(t u-u t) t \in Z$. Since $t u-u t \in Z$, we have that $(t u-u t)\left(t^{2} u-u t^{2}\right) \in Z$. However, since $t u-u t \in Z$ then $t^{2} u-u t^{2}$ $=2 t(t u-u t)$ and therefore, $2 t(t u-u t)^{2} \in Z$. Fix $t \in T$, then either $t u-u t=0$ for all $u \in U$ or there exists $u_{0} \in U$ such that $t u_{0}-u_{0} t \neq 0 \in Z$. On the one hand, if $t u_{0}-u_{0} t \neq 0 \in Z$, then $\left(t u_{0}-u_{0} t\right)^{2}$ has an inverse and since $2 t\left(t u_{0}-u_{0} t\right)^{2} \in Z$, then $t \in Z$ would follow. On the other hand, if $t u-u t=0$ for all $u \in U$, then by the argument of the previous paragraph it follows that $t^{2} \in Z$. Thus, in either case, $t^{2} \in Z$ for all $t \in T$ and, as before, $[U, U] \subset Z$.

Involution of the second kind. We are now in a position to prove our main result in the case where the involution is of the second kind.

Lemma 12. If $A$ has an involution of the second kind, and $U$ is a Lie ideal of $[K, K]$ such that $[U, U] \subset Z$ then $U \subset Z$.

Proof. Let $q \neq 0 \in Z \cap K$. Then, by I.emma $7, A=K+q K$. Therefore, $[A, A]=[K, K]+q[K, K]$. Let $U$ be a Lie ideal of $[K, K]$. Then $[Z U$, $[A, A]]=[Z U,[K, K]]+[Z U, q[K, K]]$. Thus, $[Z U,[A, A]] \subset Z U$. How- 
ever, $Z U \subset[A, A]$ since $U \subset[K, K]$. Thus, $Z U$ is a Lie ideal of $[A, A]$ and, by Baxter [1], either $Z U \subset Z$ or $Z U=[A, A]$. If, on the one hand, $Z U \subset Z$ then $U \subset Z$. If, on the other hand, $Z U=[A, A]$ then since $[Z U, Z U]=Z[U, U]$ and $[U, U] \subset Z$, we have $[Z U, Z U]=[[A, A],[A, A]] \subset Z$. By Herstein $[3]$, we have that $[[A, A],[A, A]]=[A, A]$. Thus, $[A, A] \subset Z$ and this implies that $A$ is commutative, which is a contradiction. Thus, $U \subset Z$.

THEOREM 5. If $A$ is a simple ring of characteristic not 2 , with an involution of the second kind defined on it, and if $U$ is a proper Lie ideal of $[K, K]$, then $U \subset Z$.

Proof. By Lemma 11 , either $\bar{U}=A$ or $[U, U] \subset Z$. By Lemma 12 , if $[U, U] \subset Z$ then $U \subset Z$. Thus, we must dispose of the case where $\bar{U}=A$. Define for all $n, n \geqq 1, U^{(n)}=\left[U^{(n-1)}, U^{(n-1)}\right]$ and $U^{(1)}=[U, U]$. If $U^{(n)} \subset Z$ for some $n$ then, by Lemma $12, U^{(n-1)} \subset Z$ and so, by repeated application of Lemma 12, $U \subset Z$. Thus, we can suppose that $\left[U^{(n)}\right]^{-}=A$ for all $n$. In particular, $[U, U]^{-}=A$ and therefore, by Lemma $9,[[U, U], A]=[A, A]$. Thus, by Lemma $10,[[U, U], K]=[K, K]$. But, since $[U, U] \subset T,[[U, U]$, $K] \subset U$. Hence $U \supset[K, K]$. This contradicts $U$ being a proper Lie ideal of $[K, K]$. This then completes the proof of the theorem.

Involution of the first kind. In this section we assume the involution is of the first kind. In this case $K \cap Z=(0)$ and then Lemma 11 reads:

Lemma 11b. If $U$ is a proper Lie ideal of $[K, K]$, then either $U=A$ or $[U, U]=(0)$.

The question in this case is answered by showing that $[U, U]=(0)$ implies $U=(0)$, while $\bar{U}=A$ implies that $U=[K, K]$.

Lemma 13. If $[U, U]=(0)$, then for each $u \in U$ either $u^{3}=0$ or $u$ has an inverse.

Proof. If $l \in[K, K]$ and $u \in U$, then

(I) $D_{u}^{(2)}(l)=0$.

If $s \in S$, then $\left[u^{2}, s\right] \in[K, K]$ and hence

(II) $D_{u}^{(2)}\left(\left[u^{2}, s\right]\right)=0$.

However, if $k \in K$, then $D_{u}(k) \in[K, K]$ and hence

(III) $D_{u}^{(3)}(k)=0$.

Therefore,

(IV) $u D_{u}^{(3)}(k)+D_{u}^{(3)}(k) u=0$.

This is equivalent to

(V) $D_{u}^{(2)}\left(\left[u^{2}, k\right]\right)=0$.

Hence, for all $a \in A$ we have by combining (II) and (V) that

(VI) $D_{u}^{(2)}\left(\left[u^{2}, a\right]\right)=0$.

That is, $u^{4} a-a u^{4}-2 u\left(u^{2} a-a u^{2}\right) u=0$ for all $a \in A$. Therefore, $u^{4} A \subset A u$. Multiply on the left by $A$ and we have $A u^{4} A \subset A^{2} u=A u$. Hence, since $A$ is simple, either $u^{4}=0$ or $A=A u$. On the one hand, if $u^{4} \neq 0$ then by a similar 
argument $A=u A$. Suppose $A u=u A=A$; then there exists a unit element $\rho \in A$ and $u$ has an inverse. On the other hand, $u^{4}=0$ implies by (VI) that $2 u\left(u^{2} a-a u^{2}\right) u=0$ and, multiplying on the left by $u^{2}$ and using the fact that the characteristic is not 2 , we have $u^{3} a u^{3}=0$ for all $a \in A$; that is, $u^{3} A$ is a nilpotent right ideal, which is impossible in a simple ring unless $u^{3} A=(0)$ and so $u^{3}=0$.

Lemma 14. If $[U, U]=(0)$ and $u^{3}=0$ for all $u \in U$, then $U=(0)$.

Proof. Since $[U, U]=(0)$ we have $u v=v u$ for all $u, v \in U$. Let $u \in U$, $k \in[K, K]$; then $u(u k-k u)-(u k-k u) u=0$. By multiplying this expression on the left by $u^{2}$, we have $u^{2} k u^{2}=0$ for all $u \in U, k \in[K, K]$. Let $v \in U$ and $l \in K$; then $v l-l v \in[K, K]$ and hence $u^{2}(v l-l v) u^{2}=0$. By multiplying on the left by $v^{2}$ and on the right by $v$, we have

(I) $u^{2} v^{2} l u^{2} v^{2}=0$ for all $l \in K$.

Also, $v^{2} s-s v^{2} \in[K, K]$ for all $v \in U, s \in S$ and hence $u^{2}\left(v^{2} s-s v^{2}\right) u^{2}=0$. By multiplying on the left by $v^{2}$, we then have

(II) $u^{2} v^{2} s u^{2} v^{2}=0$ for all $s \in S$.

Hence, for all $a \in A, u^{2} v^{2} a u^{2} v^{2}=0$. That is, $u^{2} v^{2} A$ is a nilpotent right ideal and this is possible only if $u^{2} v^{2}=0$ for all $u, v \in U$.

Therefore, if $u, v, w \in U$ then $0=u^{2}(v+w)^{2}=u^{2}\left[v^{2}+2 v w+w^{2}\right]=2 u^{2} v w$ and since the characteristic is not 2 , then $u^{2} v w=0$ for all $u, v, w \in U$; that is, $u^{2} v U=(0)$. Thus, by Lemma $5, u^{2} v=0$ and hence $u^{2} U=(0)$. By Lemma 5, this implies that $u^{2}=0$ for all $u \in U$. Hence, by Lemma 7, $U=(0)$. This completes the proof of the lemma.

Lemma 15. If $[U, U]=(0)$ and $N$ is the set of all nilpotent elements of $U$, then $N=(0)$.

Proof. First consider the case where the characteristic $\neq 3$. Since $[U, U]$ $=(0)$ we have, by Lemma 13 , either $u^{3}=0$ or $u$ has an inverse. Let $N$ be the set of nilpotent elements of $U$. The claim is that $N$ is closed with respect to addition. Let $u, v \in N$ and consider $u+v$. Either $(u+v)^{3}=0$ or $u+v$ has an inverse. Suppose $(u+v)$ has an inverse. Then, $(u+v)^{3}=3 u v(u+v)$ since $u v=v u$ and $u^{3}=v^{3}=0$. Hence, $(u+v)^{2}=3 u v$ and, since $(u+v)^{2}$ has an inverse, it follows that $u$ has an inverse, which is a contradiction. Thus, $(u+v)^{3}=0$. Let $t \in N$ be such that $t^{3}=0$ and $t^{2} \neq 0$. The claim is that $t k-k t \in N$ for all $k \in[K, K]$. Since $t k-k t$ commutes with $t$, then $t^{2} k-k t^{2}=2 t(t k-k t)$. Hence $t^{3} k-k t^{3}=3 t^{2}(t k-k t)$. Therefore, $0=3 t^{2}(t k-k t)$ since $t^{3}=0$. If $t k-k t$ is not nilpotent, it has an inverse which would mean $t^{2}=0$ contrary to hypothesis. So $t k-k t$ is nilpotent. If $t^{2}=0$ and $t \neq 0$ then $t^{2} k-k t^{2}=2 t(t k-k t)$. Hence, as before, $t k-k t$ must be nilpotent or else $t=0$, a contradiction. Thus, $N$ is a Lie ideal of $[K, K]$ such that every element is nilpotent of index 3. By Lemma 14 , we have $N=(0)$.

If the characteristic is 3 , we know that $u^{3} k-3 u\left(u^{2} k-k u^{2}\right) u-k u^{3}=0$ for all $u \in U, k \in K$. Thus, $u^{3} k=k u^{3}$ and therefore, $u^{3} k^{\prime}=k^{\prime} u^{3}$ for all $k^{\prime} \in \bar{K}$ which 
implies that $u^{3} \in Z$. Since $u^{3} \in K$ and $Z \cap K=(0)$, we have that $u^{3}=0$ for all $u \in U$. Thus, by Lemma $14, U=(0)$.

Note. If the involution is of the first kind, then every nonzero element of a Lie ideal $U$ of $[K, K]$ where $[U, U]=(0)$ has an inverse.

Lemma 16. If $[U, U]=(0)$ then $u^{2}$ commutes with

$$
u^{2}\left(u^{2} a-a u^{2}\right)-\left(u^{2} a-a u^{2}\right) u^{2} \text { for all } a \in A \text {. }
$$

Proof. In Lemma 13 we saw that $D_{a}\left(u^{4}\right)=2 u D_{a}\left(u^{2}\right) u$. However, $D_{a}\left(u^{4}\right)$ $=D_{a}\left(u^{2} u^{2}\right)=D_{a}\left(u^{2}\right) u^{2}+u^{2} D_{a}\left(u^{2}\right)$. Setting $D_{a}\left(u^{2}\right)=x$, we then have that

$$
x u^{2}+u^{2} x=2 u x u \text {. }
$$

That is, $D_{u}^{(2)}(x)=0$. Hence, $D_{u^{2}}^{(2)}(x)=0$. However, since $x=D_{u^{2}}(a)$ then $D_{u^{2}}^{(3)}(a)$ $=0$. That is, $u^{2}$ commutes with $D_{u^{2}}^{(2)}(a)=u^{2}\left(u^{2} a-a u^{2}\right)-\left(u^{2} a-a u^{2}\right) u^{2}$.

Lemma 17. Let $U$ be a Lie ideal of $[K, K]$ such that $[U, U]=(0)$; then $U=(0)$.

Proof. In the proof of Lemma 15, we have shown that if the characteristic is 3 then $[U, U]=(0)$ implies $U=(0)$. Therefore, assume the characteristic is not 3. Letting $w=u^{2}$ we see, by Lemma 16 , that $D_{w}^{(3)}(a)=0$ for all $a \in A$. Replacing $a$ by $a b$, we have $0=D_{w}^{(3)}(a b)=D_{w}^{(3)}(a) b+3 D_{w}^{(2)}(a) D(b)+3 D_{w}(a) D_{w}^{(2)}(b)$. $+a D_{w}^{(3)}(b)=3\left[D_{w}^{(2)}(a) D_{w}(b)+D_{w}(a) D_{w}^{(2)}(b)\right]$. Since the characteristic is not 3, $0=D_{w}^{(2)}(a) D_{w}(b)+D_{w}(a) D_{w}^{(2)}(b)$. Let $b=D_{w}(a)$; then $D_{w}^{(2)}(b)=0$ and we have

Now,

(I) $0=\left[D_{w}^{(2)}(a)\right]^{2}$.

(II) $u^{2} k^{\prime}-2 u k^{\prime} u+k^{\prime} u^{2} \in U$ for all $k^{\prime} \in K$.

Let $k^{\prime}=u k u$ in (II) and, since $[U, U]=(0)$, we have

(III) $u^{2} D_{u}^{(2)}(k) \in U$ for all $k \in K$.

Thus, since $[U, U]=(0)$, it follows that

(IV) $D_{u^{2}}^{(2)}(k)=4 u^{2} D_{u}^{(2)}(k)$ for all $k \in K$.

On the one hand by (III), $D_{u^{2}}^{(2)}(k) \in U$ and on the other hand by (I), $D_{u^{2}}^{(2)}(k)$ is nilpotent. Thus, by Lemma 15 ,

(V) $D_{u^{2}}^{(2)}(k)=0$ for all $k \in K$.

Also, setting $a=s$ in (I) we have, since $[U, U]=(0)$, that

$$
0=4 u^{2}\left[u\left(u^{2} s-s u^{2}\right)-\left(u^{2} s-s u^{2}\right) u\right]^{2}
$$

for all $s \in S$. However, we have seen that every nonzero element of $U$ has an inverse; thus $u^{2}$ has an inverse and hence,

(VI) $0=\left[u\left(u^{2} s-s u^{2}\right)-\left(u^{2} s-s u^{2}\right) u\right]^{2}$.

Since the quantity within the bracket in (VI) is in $U$ and is nilpotent, then by Theorem $15, u\left(u^{2} s-s u^{2}\right)=\left(u^{2} s-s u^{2}\right) u$ for all $s \in S$. Thus,

(VII) $D_{u^{2}}^{(2)}(s)=0$

and therefore, by combining (V) and (VII), we have for all $a \in A$

(VIII) $D_{u^{2}}^{(2)}(a)=0$. 
Hence, by Lemma $1, u^{2} \in Z$ for all $u \in U$. But, $u^{2} k-2 u k u+k u^{2}=0$ for all $k \in[K, K]$; that is, since $u^{2} \in Z, 2 u(u k-k u)=0$ and, since $u$ has an inverse, $u k=k u$ for all $k \in[K, K]$. Therefore, $u k^{\prime}=k^{\prime} u$ for all $k^{\prime} \in[K, K]-$ and, since $[K, K]-=A$, we obtain that $U \subset Z$. Since $Z \cap K=(0)$, then $U=(0)$. This completes the proof of our lemma.

We now state the main theorem concerning simple rings with involution of the first kind.

THEOREM 6. If $A$ is a simple ring of characteristic $\neq 2$, with either its center $Z=(0)$ or of dimension greater than 16 over its center, and with an involution of the first kind defined on it, then $[K, K]$ is a simple Lie ring.

Proof. By Lemma $11 \mathrm{~b}$, if $U$ is a Lie ideal of $[K, K]$ then either $[U, U]$ $=(0)$ or $\bar{U}=A$. If, on the one hand, $[U, U]=(0)$ then, by Lemma 17 , $U=(0)$. If, on the other hand, $\bar{U}=A$ then, as in the proof of Theorem 5, $U=[K, K]$. Hence, $[K, K]$ is a simple Lie ring.

Thus, by combining Theorems 5 and 6 , we are in a position to state the main result of this paper as follows:

THEOREM 7. If $A$ is a simple ring of characteristic $\neq 2$, with either its center $Z=(0)$ or of dimension greater than 16 over its center, and with an involution defined on it, then (i) $[K, K]$ is a simple Lie ring if the involution is of the first kind, and (ii) $[K, K]$ modulo its intersection with the center of $A$ is a simple Lie ring if the involution is of the second kind.

Some relationships between $S$ and $K$. Now, by using the facts that $\bar{K}=A$ and $\bar{S}=A$, we are in a position to show some interesting relations between the sets $S$ and $K$.

Notation. If $U$ and $V$ are additive subgroups of $A$, by $U \circ V$ we mean the additive subgroup of $A$ generated by all elements of form $u v+v u$ where $u \in U, v \in V$.

TheOREM 8. Let $A$ be a simple ring, of characteristic not 2 , with $Z=(0)$ or of dimension greater than 16 over $Z$, and with an involution defined on it; then $S=K \circ K$.

Proof. Let $k_{1}, k_{2} \in K$; then $k_{1} k_{2}-k_{2} k_{1} \in K$ and $k_{1} k_{2}+k_{2} k_{1} \in S$. Since $2 k_{1} k_{2}$ $=\left(k_{1} k_{2}-k_{2} k_{1}\right)+\left(k_{1} k_{2}+k_{2} k_{1}\right)$ for all $k_{1}, k_{2} \in K$, then $K^{2} \subset K+K \circ K$. Denote by $L$, the set $K+K \circ K$. Assume that for all $i \leqq n, K^{i} \subset L$. Then, $\left(k_{1} k_{2}-k_{2} k_{1}\right) k_{3} k_{4} k_{5} \cdots k_{n+1} \in L$, and $k_{2}\left(k_{1} k_{3}-k_{3} k_{1}\right) k_{4} k_{5} \cdots k_{n+1} \in L$. Thus, by adding, we have that $\left(k_{1} k_{2} k_{3}-k_{2} k_{3} k_{1}\right) k_{4} k_{5} \cdots k_{n+1} \in L$. Also, by the induction hypothesis, $\left(k_{2} k_{3} k_{1}+k_{1} k_{3} k_{2}\right) k_{4} k_{5} \cdots k_{n+1} \in L$ since $k_{2} k_{3} k_{1}+k_{1} k_{3} k_{2} \in K$ and, by adding the last two expressions, we have that $k_{1}\left(k_{2} k_{3}+k_{3} k_{2}\right) k_{4} k_{5} \cdots k_{n+1} \in L$. However, $k_{1}\left(k_{2} k_{3}-k_{3} k_{2}\right) k_{4} k_{5} \cdots k_{n+1} \in L$, and thus $2 k_{1} k_{2} k_{3} k_{4} \cdots k_{n+1} \in L$; that is, $K^{n+1} \subset L$. Thus, given $k^{\prime} \in \bar{K}$, we have that $k^{\prime} \subset K+K \circ K$; that is, $\bar{K} \subset K+K \circ K$. Hence, since $A=\bar{K}$, we have that $A=K+K \circ K$. Trivially 
$K \circ K \subset S$; however, since $A=S+K$ and $S \cap K=(0)$, we have that $S \subset K \circ K$, that is, $S=K \circ K$.

This result leads to the following interesting theorem regarding the relationship between the individual elements of $S$ and those of $K$.

Theorem 9. Let $A$ be a simple ring, of characteristic not 2, with $Z=(0)$ or of dimension greater than 16 over $Z$, and with an involution defined on it; then every symmetric element can be written as a finite sum of squares of skew elements with coefficients \pm 1 .

Proof. Since $S=K \circ K$, then every element of $S$ can be written as a finite sum of elements of the form $\{k l+k l\}$ where $k$ and $l \in K$. But $k l+l k=(k+l)^{2}$ $-k^{2}-l^{2}$. Since $k$ and $l \in K$ and since $K$ is an additive subgroup, then $k+l \in K$. Hence, our contention is verified.

Corollary. If $K$ is finite dimensional, then so is $A$.

Proof. If $K$ is of dimension $n$, then if $k_{1}, k_{2}, \cdots, k_{n}$ is a basis of $K$, $\left\{k_{i} k_{j}+k_{j} k_{i}\right\}$ spans $S$, so $k_{1} \cdots, k_{n}, k_{i} k_{j}+k_{j} k_{i}$ span $A$. Hence, $A$ is at most of dimension $n+n^{2}$.

THEOREM 10. Let $A$ be a simple ring, of characteristic not 2 , with $Z=(0)$ or of dimension greater than 16 over $Z$, and with an involution defined on it; then $S \circ K=K$; that is, the additive subgroup generated by all elements of form $s k+k s, s \in S, k \in K$ is $K$.

Proof. We first claim that $S \circ K \supset[K, K]$. Let $s \in S, k_{1}, k_{2} \in K$; then $\left(s k_{1}+k_{1} s\right) k_{2}-k_{2}\left(s k_{1}+k_{1} s\right)=\left\{s\left(k_{1} k_{2}-k_{2} k_{1}\right)+\left(k_{1} k_{2}-k_{2} k_{1}\right) s\right\}+\left\{k_{1}\left(s k_{2}-k_{2} s\right)\right.$ $\left.+\left(s k_{2}-k_{2} s\right) k_{1}\right\}$. The first \{\} is in $S \circ K$ since $k_{1} k_{2}-k_{2} k_{1} \in K$, and the second \{\} is in $S \circ K$ since $s k_{2}-k_{2} s \in S$. Therefore, $[S \circ K, K] \subset S \circ K$. Hence, by Theorem 1, $S \circ K \supset[K, K]$ or $S \circ K \subset Z$. If $S \circ K \subset Z$, then $s(s k+k s)$ $=(s k+k s) s$. Therefore, $s^{2} k=k s^{2}$ and so $s^{2} \in Z$ since $\bar{K}=A$. Likewise, $k(s k+k s)$ $=(s k+k s) k$. Therefore, $k^{2} s=s k^{2}$ and so $k^{2} \in Z$ since $\bar{S}=A$. If $a \in A, a=s+k$, then $a^{2}=s^{2}+s k+k s+k^{2} \in Z$. This forces $A$ to be commutative; thus $S \circ K$ $\supset[K, K]$.

Using this fact, we shall now show that $S \circ K=K$. Let $s_{1}, s_{2} \in S$; then $2 s_{1} s_{2}=\left(s_{1} s_{2}+s_{2} s_{1}\right)+\left(s_{1} s_{2}-s_{2} s_{1}\right)$. Since $s_{1} s_{2}+s_{2} s_{1} \in S$ and $s_{1} s_{2}-s_{2} s_{1} \in[S, S]$, we have $S^{2} \subset S+[S, S]$. However, by Lemma $10,[S, S]=[K, K]$. Therefore, since $[K, K] \subset S \circ K$, then $S^{2} \subset S+S \circ K$. Also, if $s \in S$ and $k \in K$, then $2 s k=(s k+k s)+(s k-k s)$ and, since $s k+k s \in S \circ K$ and $s k-k s \in S$, then $S K \subset S+S \circ K$. Thus,

(I) $S A \subset S+S \circ K$.

Multiplying (I) on the left by $S$, we have $S^{2} A \subset S^{2}+S K \subset S+S \circ K$. Continuing in this manner, $S^{n} A \subset S+S \circ K$. Thus, $\bar{S} A \subset S+S \circ K$ and, since $\bar{S}=A$, $A^{2} \subset S+S \circ K$. Since $A$ is simple, $A^{2}=A$ and thus $A \subset S+S \circ K$. However, since $S \cap K=(0)$, then $S \circ K=K$. 


\section{REFERENCES}

1. W. E. Baxter, Lie simplicity of a special class of associative rings, Proc. Amer. Math. Soc. vol. 7 (1956) pp. 855-863.

2. I. N. Herstein, On the Lie and Jordan rings of a simple associative ring, Amer. J. Math. vol. 77 (1955) pp. 393-403.

3. —- The Lie ring of a simple associative ring, Duke Math. J. vol. 22 (1955) pp. 471476.

4. - Lie and Jordan systems in simple rings with involution, Amer. J. Math. vol. 78 (1956) pp. 629-649.

5. I. Kaplansky, Rings with a polynomial identity, Bull. Amer. Math. Soc. vol. 54 (1948) pp. 575-580.

University of Pennsylvania

Philadelphia, Pa. 